\title{
Consumo de fármacos opiáceos en la ciudad de Madrid: Factores sanitarios y sociodemográficos asociados
}

\section{Opiate drug use in the city of Madrid:} Associated health and sociodemographic factors

\author{
Eduardo J. Pedrero-Pérez*, Sara Morales-Alonso*, Blanca Álvarez-Crespo*, \\ María Teresa Benítez-Robredo*. \\ * Unidad de Formación e Investigación. Departamento de Evaluación y Calidad. Madrid Salud. Ayuntamiento de Madrid.
}

\section{Resumen}

El consumo de analgésicos opiáceos ha provocado una situación de emergencia sanitaria y social en Estados Unidos. En España, según datos oficiales, la prescripción de estos fármacos ha experimentado un espectacular ascenso en la última década. Este estudio explora la prevalencia del uso de estos fármacos y las características sanitarias y sociodemográficas de sus consumidores en la ciudad de Madrid. Se realizó una encuesta telefónica aplicando un muestreo estratificado y aleatorizado, en la que se preguntó por el uso de estos fármacos y si fueron médicamente prescritos o no. La muestra estuvo compuesta por $\mathrm{n}=8.845$ sujetos de edades entre 15 y 98 años. Un $16,0 \%$ declara haber usado estos fármacos en el último año y un 9,1 los toma en las dos últimas semanas. El consumo es más frecuente en mujeres, clase social baja y menor nivel de estudios. El grupo más joven (15-29 años) ya lo usa en el 12,5\%. Quienes usan opioides refieren peor salud percibida, menor calidad de vida, más problemas de salud mental, más soledad no deseada, más uso de otros psicofármacos, más frecuente uso diario de tabaco y menos consumo problemático de alcohol. Un 10\% de quienes los usan lo hacen sin prescripción médica. Combinando estos datos con los de prescripción ofrecidos por el Ministerio de Sanidad, resulta necesario prestar atención a un problema que puede hacerse patente en los próximos años, aconsejando la adopción de medidas urgentes para atajarlo antes de que aproxime la situación española a la ya bien conocida en otros países.

Palabras clave: Opiáceos; adicción; encuesta de salud; salud mental; psicofármacos.

\begin{abstract}
The use of opiate analgesics has led to a health and social emergency in the United States. In Spain, according to official data, the prescription of these drugs has risen dramatically in the last decade. This study explores the prevalence of the use of these drugs and the health and socio-demographic characteristics of their consumers in the city of Madrid. A telephone survey was carried on a stratified, randomised sample, asking about the use of these drugs and whether or not they were medically prescribed. The sample consisted of $n=8,845$ subjects aged between 15 and 98 years. Sixteen percent stated that they had used these drugs in the last year and $9.1 \%$ had taken them in the last two weeks. Consumption was more frequent among women, lower social class and lower level of education. Among the youngest group (1529 years old) $12.5 \%$ had already used it. Those who use opioids report worse perceived health, lower quality of life, more mental health problems, more loneliness, more use of other psychoactive drugs, more frequent daily use of tobacco and less problematic consumption of alcohol. Ten percent of those who use them do so without a doctor's prescription. Combining these data with the prescription data offered by the Ministry of Health, it is necessary to pay attention to a problem that may become apparent in the coming years, and the adoption of urgent measures to tackle it before it brings the Spanish situation closer to that already well known in countries of our socio-political environment is advised.
\end{abstract}

Keywords: Opioids; addiction; health survey; mental health; psychopharmaceuticals.

Recibido: Abril 2019; Aceptado: Junio 2019.

Enviar correspondencia a: Eduardo J. Pedrero Pérez.

Unidad de Formación e Investigación. Dpto. de Evaluación y Calidad. Madrid Salud. Ay. de Madrid. Av. del Mediterráneo 62. 28007 Madrid. España E-mail: pedreropej@madrid.es / ejpedrero@yahoo.es 


\section{Introducción}

$\mathrm{L}$ os opiáceos, tanto los prescritos como los que se obtienen de manera ilícita, se han convertido en un importante problema de salud en los últimos años. Se suele hablar indistintamente de opiáceos y opioides, aunque los primeros son derivados naturales, mientras que los opioides son sintéticos. Ambos se utilizan generalmente para problemas relacionados con el dolor, aunque algunos también se utilizan para el tratamiento de personas con adicción, como la metadona.

El dolor se define como una experiencia sensorial o emocional desagradable, resultante de un daño tisular real o potencial. Es un importante problema de salud y una de las principales causas de consulta médica. Se define como dolor crónico aquel cuya duración es superior a 3 meses o mayor de la esperada tras la cicatrización o curación de la enfermedad subyacente (Merskey, 1986). El tratamiento del dolor crónico comprende estrategias tanto farmacológicas como no farmacológicas. Dentro de las primeras se encuentran los analgésicos no opioides, opioides y coadyuvantes (utilizados para prevenir o tratar los efectos secundarios de los opioides o potenciar la analgesia). Los fármacos opioides constituyen un grupo de fármacos que se caracterizan por poseer afinidad selectiva por los receptores opioides centrales y periféricos, inhibiendo la transmisión de la entrada nociceptiva y la percepción del dolor (Rosenquist, 2019). Son ampliamente aceptados para el tratamiento del dolor agudo severo y del dolor crónico de moderado a severo que no responde a otros tratamientos (Dowell, Haegerich y Chou, 2016).

El uso de este tipo de fármacos conlleva una serie de riesgos. Una investigación reciente (Gaspari et al., 2014) revela que el consumo de sustancias opioides altera la actividad de una proteína específica necesaria para el funcionamiento normal del centro de recompensa del cerebro. Según los autores, como el centro de recompensa del cerebro tiene un impacto tan fuerte sobre las respuestas analgésicas, deberían utilizarse medicamentos no opioides para el tratamiento del dolor crónico intenso. Además, los opioides favorecen neuroadaptaciones que disminuyen su capacidad para proporcionar analgesia a medio y largo plazo, produciendo efectos opuestos, es decir, el aumento del dolor existente (hiperalgesia inducida por opioides) y la facilitación del desarrollo del dolor crónico (Lavand'homme y Steyaert, 2017). Otros estudios enumeran complicaciones del uso de opiáceos para el dolor crónico no oncológico, que deben ser tenidas en cuenta (Els et al., 2017). Por estas razones y por la limitada utilidad de los opioides en el tratamiento del dolor crónico no se recomienda en la actualidad su uso extrahospitalario, mucho menos en dolores moderados o leves (Ashburn y Fleisher, 2018).

El uso indiscriminado de estos medicamentos está generando un importante problema de salud en países desarrollados, como la denominada epidemia de opiáceos en
Estados Unidos (EEUU) más de 250.000 muertos (Guardia Serecigni, 2018; Marshall, Bland, Hulla y Gatchel, 2019; Skolnick, 2018). Esta epidemia y todas sus consecuencias no pueden ser explicadas sólo mediante propiedades farmacológicas de las sustancias, sino que responden a muy diferentes circunstancias psicosociales, culturales y económicas, que no siempre se han tenido en cuenta (Kolodny et al., 2015). Recientes estudios encuentran una correlación positiva entre el gasto farmacéutico en promoción de estos analgésicos opioides con el número de muertes en diferentes zonas del país (Hadland, Cerdá, Li, Krieger y Marshall, 2018; Hadland, Rivera-Aguirre, Marshall y Cerdá, 2019).

En España, hasta 1997, la morfina era prácticamente el único opioide de tercer escalón prescrito (94\% del total), pero desde la aparición del fentanilo en la farmacopea española el incremento en el uso de este fármaco ha sido espectacular. Desde 1997 hasta 2001, la prescripción de opiáceos se duplicó en Madrid (Alonso Babarro, Varela Cerdeira y Aparicio Jabalquinto, 2003), para triplicarse después entre 2004 y 2011 (Ruiz-López y Alonso-Babarro, 2019). Atendiendo a los años más recientes, los principios activos opioides más prescritos en España para el tratamiento extrahospitalario del dolor han sido el tramadol (62,2\% de los opioides), el fentanilo (17,5\%) y la buprenorfina $(6,9 \%)$, datos obtenidos a partir de los fármacos dispensados con cargo a receta del Sistema Nacional de Salud (AEMPS, 2017). En el reciente informe de la Agencia Española del Medicamento y Productos Sanitarios (AEMPS, 2019), se constata el crecimiento constante del uso de este tipo de fármacos, que desde el 2010 han alcanzado en 2017 el 179\% en cuanto a dosis humanas diarias (DHD) prescritas con receta (Figura 1). En cuanto al fentanilo en particular, el incremento desde 2008 se sitúa en el $185 \%$. El aumento de prescripción y uso de este fármaco ha resultado exponencial, de modo que, según datos del Pain \& Policy Studies Group de la Universidad de Wisconsin, España ocupaba en el año 2000 el puesto $15^{\circ}$ en volumen de consumo de fentanilo, habiendo pasado al $5^{\circ}$ en 2014 (Calabozo Freile, 2017).

El caso del tramadol es especial, puesto que actualmente llega a ser prescrito como fármaco de primera elección para dolor leve y puede obtenerse incluso sin receta, dado que se considera un "opioide débil"; no obstante, este fármaco se vincula con muerte prematura, ya sea por su capacidad adictiva o por sus interacciones con otros fármacos (Randall y Crane, 2014). En la Figura 2, y según datos de la AEMPS (2019), la prescripción de tramadol, en cualquiera de sus presentaciones, se ha triplicado entre 2010 y 2017.

El último estudio EDADES del Plan Nacional sobre Drogas (DGPNSD, 2018) incluye por primera vez preguntas que exploran esta cuestión en el conjunto de la población española. Aunque la situación en España está lejos de las cifras de EEUU, el incremento de las prescripciones y el aumento 


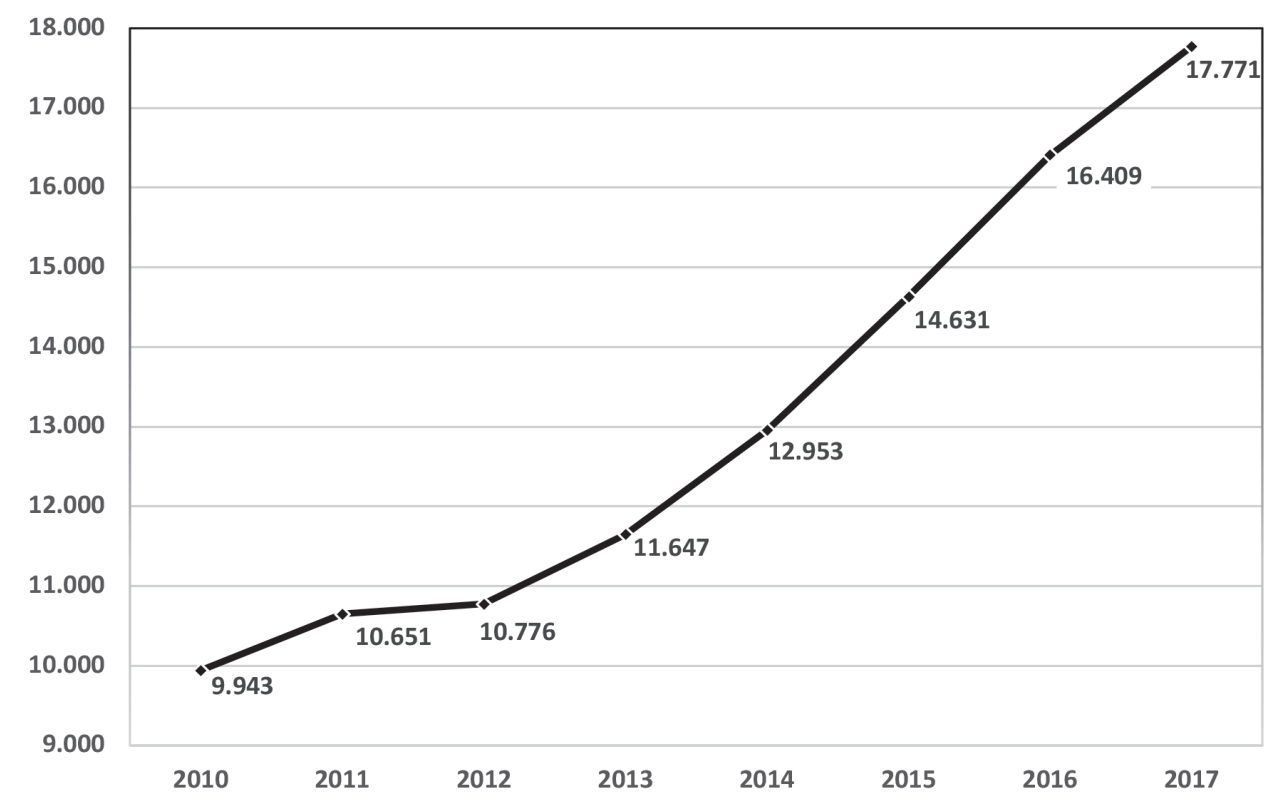

Figura 1. Dosis humanas diarias (DHD), prescritas con receta, de fármacos opiáceos en España. FUENTE: AEMPS, 2019.

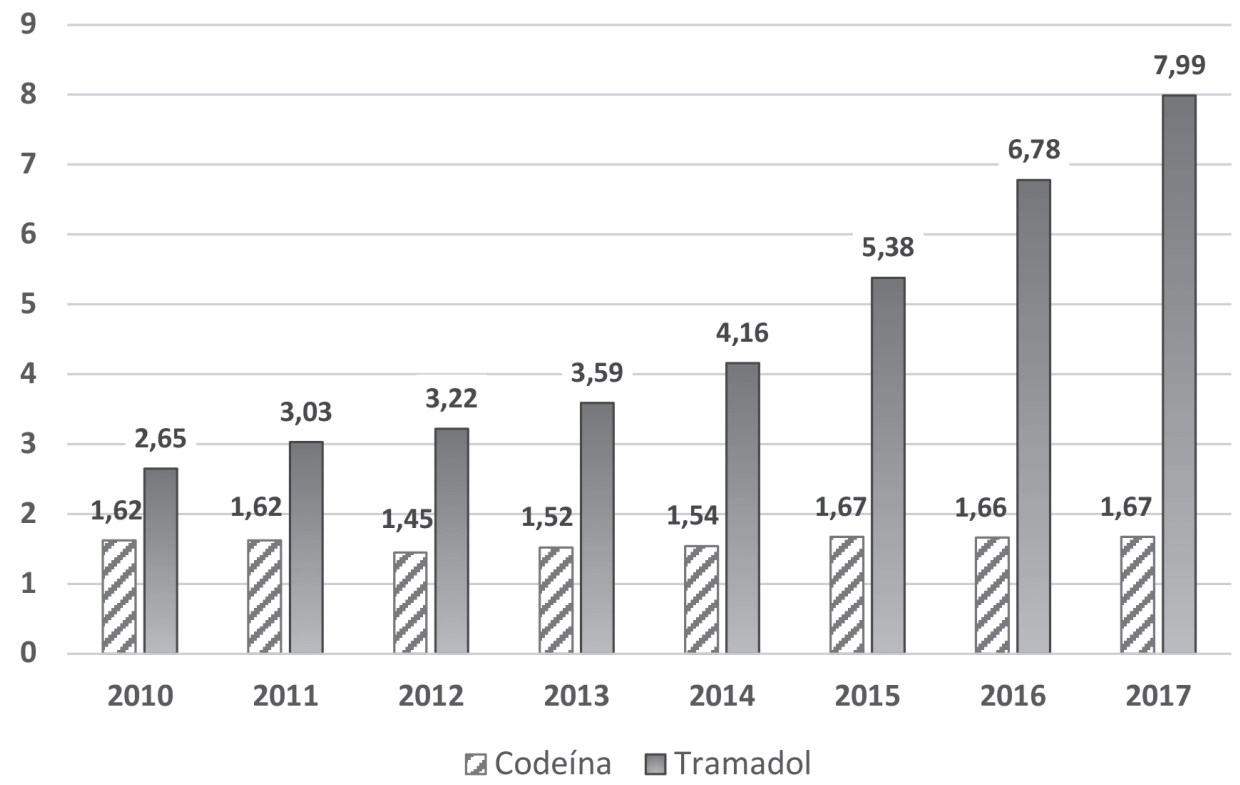

Figura 2. Dosis humanas diarias (DHD) de codeína (con ibuprofeno o paracetamol) y tramadol (con paracetamol y, en el último año, con dexketoprofeno) prescritas en España. FUENTE: modificado de AEMPS, 2019.

del número de casos de adicción en los últimos años ha llevado a varias organizaciones científicas a elaborar una Guía para el buen uso de analgésicos opioides (Socidrogalcohol, 2017), en la misma línea en que ya han sido escritas en otros países (Busse et al., 2017; Dowell et al., 2016).

Todos los datos disponibles se han obtenido a partir de los medicamentos prescritos, sin que sea posible incluir el uso no prescrito o procedente del mercado gris o directamente del mercado negro (sin olvidar la posible obtención de fármacos por Internet). Ninguno de estos informes explora el consumo individual de estos fármacos, las razones para su uso o las características de quienes los utilizan. Este estudio trata de conocer el consumo actual de este tipo de medicamentos en la población de la ciudad de Madrid y su asociación con variables sociodemográficas e indicadores de salud, así como obtener indicios sobre la forma de obtención y consumo: prescripción médica o autoadministración. 


\section{Material y Método}

\section{Muestra y procedimiento}

La población sobre la que se realizó la encuesta la componían las personas residentes en la ciudad de Madrid de 15 y más años de edad. A partir del padrón municipal de 2017 se estimó una muestra representativa del conjunto de la ciudad y de sus distritos, con un error muestral inferior al $1 \%$ para un nivel de confianza del $95 \%$ en estimaciones correspondientes a categorías equiprobables $(\mathrm{p}=\mathrm{q}=0,5)$ y en el supuesto de muestreo aleatorio simple. Se diseñó un muestreo aleatorio estratificado, teniendo como criterio de estratificación los 21 distritos de la ciudad, estableciendo como mínimo un $\mathrm{n}=400$ en cada distrito, $\mathrm{y}$, como segundo paso, una posestratificación por sexo y grupo de edad a partir de tamaños mínimos fijados de antemano. De este modo, se aseguró un error de muestreo en distritos menor del $5 \%$ en las mismas condiciones que las definidas. Se realizaron 9.676 entrevistas telefónicas, de las que 8.845 pudieron completarse y se consideraron válidas (ver descriptivos en Tabla 1). La selección de las personas a entrevistar en cada estrato se realizó aleatoriamente en los hogares, mediante llamadas a teléfonos fijos y a móviles. La muestra se obtuvo en dos etapas: la primera fue en el hogar, al que se accede por llamada telefónica a números escogidos aleatoriamente sobre una base de datos de teléfonos fijos clasificados según los diferentes distritos de la ciudad y la segunda en que se selecciona a la persona a entrevistar entre los miembros del hogar hasta completar cuotas de edad y sexo (posestratificación) determinando una única entrevista por hogar. E1 50\% de las llamadas se realizaron a teléfonos móviles siguiendo el mismo procedimiento si bien en este caso previamente no se conocía la ubicación territorial del hogar de la persona que contestaba la llamada. El reclutamiento final contó con aproximadamente un $70 \%$ de personas conectadas a partir de un teléfono fijo. La entrevista telefónica fue realizada por encuestadores formados previamente y asistida por ordenador mediante la técnica

Tabla 1. Descriptivos de la muestra.

\begin{tabular}{lccc}
\hline \multicolumn{1}{c}{$\mathbf{n}$} & $\begin{array}{c}\text { Hombres } \\
\mathbf{4 0 5 5}\end{array}$ & $\begin{array}{c}\text { Mujeres } \\
\mathbf{4 7 9 0}\end{array}$ & $\begin{array}{c}\text { Total } \\
\mathbf{8 8 4 5}\end{array}$ \\
\hline Edad (\%) & & & \\
$15-29$ & 18,7 & 16,3 & 17,4 \\
$30-44$ & 28,6 & 25,6 & 26,9 \\
$45-64$ & 32,4 & 31,5 & 31,9 \\
$>64$ & 20,3 & 26,7 & 23,8 \\
\hline Estudios & & & \\
Primarios o menos & 7,9 & 13,2 & 10,8 \\
Secundarios & 48,3 & 46,6 & 47,3 \\
Universitarios & 43,9 & 40,2 & 41,9 \\
\hline Clase social & & & \\
Favorecida & 42,2 & 37,1 & 39,4 \\
Media & 23,3 & 25,3 & 24,4 \\
Desfavorecida & 34,5 & 37,6 & 36,2 \\
\hline
\end{tabular}

CATI (Computer Assisted Telephone Interviewing ${ }^{7}$ ) a partir de un cuestionario estructurado. El trabajo de campo se realizó entre octubre y diciembre de 2017.

El nivel relativo de desarrollo de los distritos se obtuvo mediante el cálculo del Índice Combinado de Salud, Conocimiento y Renta (ICSCR; Díaz Olalla y Benítez Robredo, 2015; pp. 200-201), un índice compuesto que se obtiene a partir de tres indicadores: (1) salud: esperanza de vida al nacer (para este estudio se utilizaron los valores de 2016); (2) educación: población de 30 a 64 años con nivel educativo superior a secundario (datos de 2017), y (3) renta: Renta Bruta Disponible per cápita (datos disponibles: 2014). Una vez obtenido el índice se efectuó un análisis de conglomerados que agrupó los distritos en cuatro categorías, que fueron denominadas: alto desarrollo, desarrollo medio alto, desarrollo medio bajo y bajo desarrollo.

Para la caracterización según clase social se clasificó a los encuestados por su clase ocupacional, siguiendo las recomendaciones de la Sociedad Española de Epidemiología para las investigaciones de salud (Domingo-Salvany et al., 2013). Se asignó a cada entrevistado la Clase Social del Hogar en que está incluido, que no necesariamente es la de la persona que contesta al cuestionario, sino la de la persona sustentadora principal de ese hogar.

En la encuesta se incluyó la pregunta: “A continuación voy a leerle una lista de tipos de medicamentos, por favor dígame si los ha consumido". Entre los medicamentos se incluían "Medicamentos fuertes para el dolor", facilitando como ejemplo una lista de los más utilizados: "tramadol, adolonta, dolantina, pazital, codeina, parches de morfina, etc.". En concreto se preguntaba: (a) si lo ha tomado en las 2 últimas semanas, en el último año -pero no en las dos últimas semanas- o si no lo ha tomado en el último año; (b) si lo ha tomado siempre prescrito por el médico o a veces sin prescripción. Preguntas similares se realizaron para fármacos antidepresivos y ansiolíticos/inductores del sueño.

El COOP/WONCA es un instrumento para estimar la calidad de vida relacionada con la salud (CVRS). Se utilizó la versión adaptada al español de Lizán y Reig (1999). Los ítems exploran aspectos relativos a esta variable a través de unas láminas en las que se visualizan mediante dibujos las 5 opciones de respuestas, debiendo marcar el evaluado la que mejor defina su estado. Aunque se han utilizado versiones de 6, 7 y 9 ítems, para el presente estudio se optó por la versión más amplia, de 9 ítems. En el estudio actual, se utilizaron los contenidos de cada ítem como estímulos verbales, del mismo modo que las respuestas, esto es, prescindiéndose de las láminas, y mediante entrevista telefónica, existiendo estudios previos que garantizan los buenos resultados de esta modalidad de administración (Pedrero-Pérez y Díaz-Olalla, 2016). Las puntuaciones se trataron como una escala Likert de 5 anclajes, correspondiendo mayores puntuaciones a peor calidad de vida. 
Cuestionario General de Salud, versión de 12 ítems ( $G \ell^{-}$ neral Health Questionnaire, GHQ-12; Goldberg y Williams, 1998), versión española (Rocha, Pérez, Rodríguez-Sanz, Borrell y Obiols, 2011) es un instrumento autoadministrado de cribado que tiene por objetivo detectar indicadores de malestar psicológico y posibles casos de trastornos psicopatológicos en contextos como la atención primaria o en población general. Los ítems se responden en una escala tipo Likert de cuatro opciones. La corrección puede efectuarse de varias formas, habiéndose adoptado en el presente estudio la opción GHQ-Likert, puntuaciones entre 0 y 3, donde las puntuaciones mayores corresponden a peores indicadores de salud, oscilando la prueba entre 0 y 36 puntos.

Se proporcionó un listado de enfermedades y otros problemas de salud, solicitando que cada participante especificara si había sido diagnosticado de cada una de ellas. Se preguntó "En los últimos doce meses, ¿̇diría que su estado de salud ha sido muy bueno, bueno, regular, malo, muy malo?' y “Con qué frecuencia Vd Se ha sentido solo/a durante el último año?'. Se preguntó si fumaban a diario. Se calculó el nivel de actividad física diaria calculando la tasa de gasto metabólico (METs) según las fórmulas de Ainsworth y cols. (2000). Se obtuvieron también variables demográficas (sexo, edad, nivel de estudios y clase social).

\section{Análisis de datos}

Para comparación de categorías se utilizó la prueba de ji cuadrado $\left(\chi^{2}\right)$, haciendo constar en subíndice los grados de libertad de cada comparación. Para estimar el tamaño del efecto se utilizó la V de Cramer. Para la comparación de variables continuas se utilizó el análisis de covarianza uni o multivariante y la omega al cuadrado $\left(\omega^{2}\right)$ como estimador del tamaño del efecto de las diferencias. Para estos análisis se utilizó el SPSS 17, calculando manualmente la $\omega^{2}$ a partir de la tabla del ANOVA.

\section{Resultados}

En la Tabla 2 se resumen los valores de las variables objeto de estudio.

Un 16,0\% (IC95\% 15,0 - 17,0) de los encuestados declara haber tomado en algún momento del año previo algún analgésico opioide. De entre ellos, un 9,1\% (IC95\% 8,3-9,9) los ha tomado en las dos últimas semanas. De entre quienes han tomado fármacos opioides en el último año la proporción de mujeres es significativamente mayor que la de hombres $\left(19,0 \%\right.$ vs. $\left.12,4 \% ; \chi_{1}^{2}=72,5 ; \mathrm{p}<0,001 ; \mathrm{V}=0,09\right)$, al igual que cuando sólo se considera el consumo en las dos últimas semanas $\left(11,9 \%\right.$ vs. $\left.5,8 \% ; \chi_{1}^{2}=98,6 ; p<0,001 ; V=0,11\right)$. En la Figura 3 se observan las frecuencias de uso de opiáceos en el último año por sexo y grupo de edad.

El consumo de opiáceos es más frecuente entre quienes tienen estudios primarios o menos (24,1\%; IC95\% 20,5 - 27,7), que entre quienes tienen estudios secundarios
(17,6\%; IC95\% 16,1 - 19,1) o universitarios (12,1\%; IC95\% $10,7-13,5)$, lo que representa diferencias significativas $\left(\chi_{2}^{2}=\right.$ 95,7; $\mathrm{p}<0,001 ; \mathrm{V}=0,10)$. También es más frecuente entre las personas de clase social desfavorecida (20,0\%; IC95\% 18,2 - 21,8) que entre las de clase media (15,0\%; IC95\% 13,0 $17,0)$ y las de clase favorecida $(13,0 \%$; IC95\% 11,4 - 14,6), diferencias también significativas $\left(\chi_{2}^{2}=61,3 ; \mathrm{p}<0,001 ; \mathrm{V}=\right.$ $0,08)$.

Cuando se les formuló la pregunta sobre la percepción del estado de salud, contestaron que era buena o muy bue-

Tabla 2. Resumen de las variables del estudio.

\begin{tabular}{|c|c|c|c|}
\hline & \multicolumn{2}{|c|}{ Han consumido opiáceos } & \multirow[b]{2}{*}{$\begin{array}{c}\text { No han } \\
\text { consumido } \\
\text { opiáceos }\end{array}$} \\
\hline & $\begin{array}{l}\text { En el último } \\
\text { año, pero no en } \\
\text { las últimas } 2 \\
\text { semanas }\end{array}$ & $\begin{array}{c}\text { En las } 2 \\
\text { últimas } \\
\text { semanas }\end{array}$ & \\
\hline \multicolumn{4}{|l|}{ Sexo } \\
\hline Hombres & $7,0 \%$ & $5,8 \%$ & $87,2 \%$ \\
\hline Mujeres & $8,1 \%$ & $11,9 \%$ & $80,0 \%$ \\
\hline \multicolumn{4}{|l|}{ Edad } \\
\hline Media & 47,6 & 53,4 & 49,1 \\
\hline D.T. & 16,4 & 17,9 & 17,9 \\
\hline \multicolumn{4}{|l|}{ Nivel de estudios } \\
\hline Primarios o menos & $11,2 \%$ & $20,0 \%$ & $9,7 \%$ \\
\hline Secundarios & $50,0 \%$ & $53,7 \%$ & $46,4 \%$ \\
\hline Universitarios & $38,8 \%$ & $26,4 \%$ & $43,8 \%$ \\
\hline \multicolumn{4}{|l|}{ Clase social } \\
\hline Favorecida & $40,0 \%$ & $26,0 \%$ & $40,9 \%$ \\
\hline Media & $23,6 \%$ & $22,4 \%$ & $24,7 \%$ \\
\hline Desfavorecida & $36,5 \%$ & $51,7 \%$ & $34,5 \%$ \\
\hline \multicolumn{4}{|l|}{$\begin{array}{l}\text { Autopercepción de } \\
\text { Salud }\end{array}$} \\
\hline Buena o Muy buena & $55,8 \%$ & $34,1 \%$ & $77,9 \%$ \\
\hline $\begin{array}{l}\text { Regular/Mala/Muy } \\
\text { mala }\end{array}$ & $44,2 \%$ & $65,9 \%$ & $22,1 \%$ \\
\hline \multicolumn{4}{|l|}{$\begin{array}{l}\text { Calidad de vida } \\
\text { (WONCA) }\end{array}$} \\
\hline Media & 2,84 & 3,35 & 2,42 \\
\hline D.T. & 0,87 & 0,73 & 0,80 \\
\hline \multicolumn{4}{|l|}{$\begin{array}{l}\text { Salud mental } \\
\text { (GHQ-12) }\end{array}$} \\
\hline Media & 11,40 & 12,50 & 9,37 \\
\hline D.T. & 5,93 & 6,29 & 4,34 \\
\hline $\begin{array}{l}\text { Riesgo de mala salud } \\
\text { mental }\end{array}$ & $33,2 \%$ & $40,2 \%$ & $17,9 \%$ \\
\hline \multicolumn{4}{|l|}{ Soledad } \\
\hline $\begin{array}{l}\text { Siempre o bastantes } \\
\text { veces }\end{array}$ & $15,5 \%$ & $21,0 \%$ & $8,7 \%$ \\
\hline \multicolumn{4}{|l|}{ Nivel de actividad } \\
\hline Alto & $43,0 \%$ & $40,2 \%$ & $43,5 \%$ \\
\hline Moderado & $46,1 \%$ & $43,4 \%$ & $44,4 \%$ \\
\hline Bajo & $10,9 \%$ & $16,5 \%$ & $12,1 \%$ \\
\hline \multicolumn{4}{|l|}{$\begin{array}{l}\text { Îndice de masa } \\
\text { corporal }\end{array}$} \\
\hline Infrapeso & $2,9 \%$ & $1,8 \%$ & $2,6 \%$ \\
\hline Normopeso & $47,5 \%$ & $40,5 \%$ & $52,1 \%$ \\
\hline Sobrepeso & $35,3 \%$ & $35,5 \%$ & $34,6 \%$ \\
\hline Obesidad & $14,4 \%$ & $22,2 \%$ & $10,8 \%$ \\
\hline
\end{tabular}




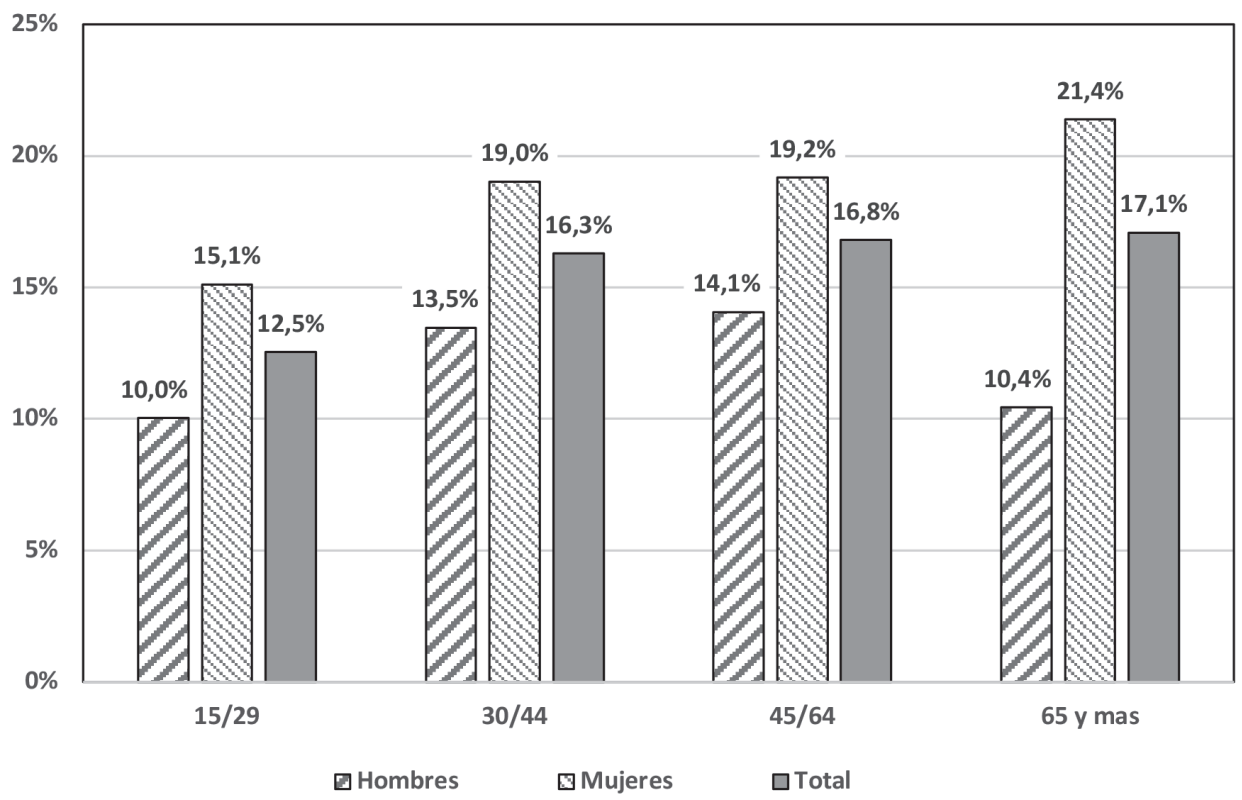

Figura 3. Frecuencia de uso de opiáceos en el último año por sexo y grupo de edad.

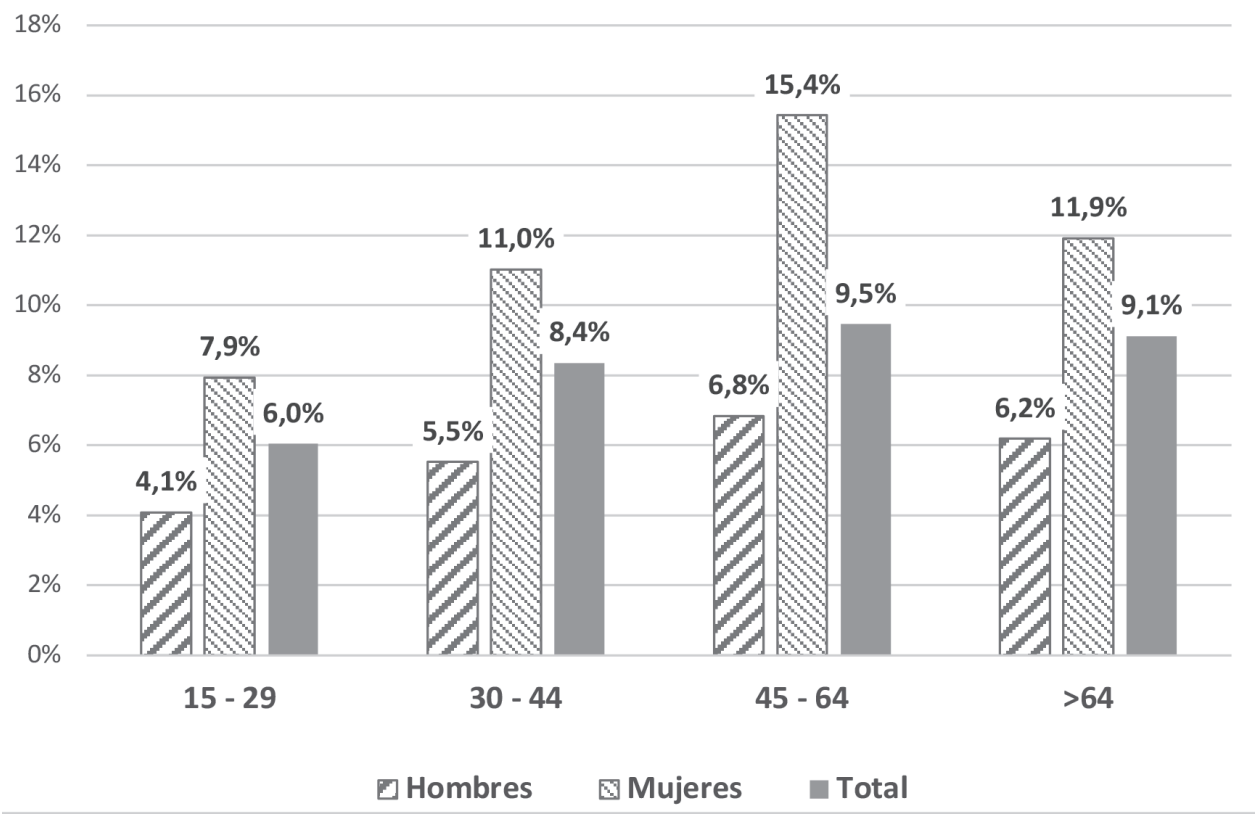

Figura 4. Consumo de analgésicos opiáceos en las dos últimas semanas por sexo y grupo de edad.

na el $43,4 \%$ de quienes tomaron medicamentos opioides frente al $77,9 \%$ de quienes no lo hicieron, lo que representa diferencias significativas y con un considerable tamaño del efecto $\left(\chi_{1}^{2}=848,1 ; p<0,001 ; V=0,31\right)$. A la pregunta sobre soledad no deseada, respondieron que siempre o casi siempre el 15,5\% de quienes toman estos fármacos, frente al 9,8\% de quienes no los toman, lo que representa diferencias significativas $\left(\chi_{1}^{2}=23,0 ; \mathrm{p}<0,001 ; \mathrm{V}=0,05\right)$.

Considerando únicamente a las personas que estaban tomando opioides en el momento de la encuesta, se observa (Figura 4) que el porcentaje de mujeres dobla al de hombres en todos los grupos de edad. Quienes han tomado analgésicos opioides en las dos últimas semanas declaran más problemas de salud mental en el GHQ-12 (M=12,5; $\mathrm{DT}=6,3)$ que quienes no los están tomando $(\mathrm{M}=9,5 ; \mathrm{DT}=$ $4,5)$ lo que representa diferencias significativas $\left(\mathrm{F}_{1}=302,9\right.$; $\left.\mathrm{p}<0,001 ; \omega^{2}=0,03\right)$. También declararon peor calidad de vida relacionada con la salud (WONCA: $\mathrm{M}=25,9 ; \mathrm{DT}=5,9$ vs. $\left.\mathrm{M}=19,6 ; \mathrm{DT}=4,7 ; \mathrm{F}_{1}=1305,2 ; \mathrm{p}<0,001 ; \omega^{2}=0,12\right)$.

El $14,1 \%$ de quienes están tomando analgésicos opioides toma también antidepresivos, frente al 4,9\% de quienes no toman aquellos $\left(\chi^{2}=115,3 ; \mathrm{p}<0,001 ; \mathrm{V}=0,11\right)$ y también están tomando más ansiolíticos $(30,4 \%$ vs. $10,1 \%$; $\left.\chi^{2}=284,8 ; p<0,001 ; \mathrm{V}=0,18\right)$. En cuanto a los diagnósticos recibidos por quienes están tomando analgésicos opioides en las dos últimas semanas, se muestra en la Tabla 3. 
Tabla 3. Porcentaje de sujetos que han sido diagnosticados de problemas de salud, según estén tomando o no analgésicos opiáceos en las dos últimas semanas.

\begin{tabular}{lcccc}
\hline Diagnóstico & $\begin{array}{c}\text { No ha } \\
\text { tomado } \\
\text { opiáceos }\end{array}$ & $\begin{array}{c}\text { Ha tomado } \\
\text { opiáceos }\end{array}$ & $\boldsymbol{\chi}_{\mathbf{1}}^{\mathbf{2}}$ & $\mathbf{V}$ \\
\hline Hipertensión & $17,7 \%$ & $29,7 \%$ & $69,2^{*}$ & 0,08 \\
Cardiopatía & $3,8 \%$ & $6,8 \%$ & 17,7 & 0,04 \\
Artrosis & $14,6 \%$ & $40,5 \%$ & $350,0^{*}$ & 0,19 \\
Dolor (cervical o dorsal) & $13,2 \%$ & $43,4 \%$ & $495,8^{*}$ & 0,23 \\
Dolor (lumbar) & $15,2 \%$ & $51,7 \%$ & $645,3^{*}$ & 0,27 \\
Alergia crónica & $21,6 \%$ & $27,3 \%$ & 13,7 & 0,03 \\
Asma & $7,9 \%$ & $12,1 \%$ & 17,6 & 0,04 \\
Bronquitis, EPOC. & $2,7 \%$ & $6,7 \%$ & $37,8^{*}$ & 0,06 \\
Diabetes & $5,5 \%$ & $11,0 \%$ & $39,2^{*}$ & 0,06 \\
Úlcera gástrica & $2,0 \%$ & $6,2 \%$ & $54,6^{*}$ & 0,07 \\
Hipercolesterolemia & $20,1 \%$ & $30,5 \%$ & $47,1^{*}$ & 0,07 \\
Depresión & $6,5 \%$ & $20,4 \%$ & $197,4^{*}$ & 0,14 \\
Ansiedad & $5,3 \%$ & $15,7 \%$ & $137,4^{*}$ & 0,12 \\
Migraña & $9,8 \%$ & $30,5 \%$ & $303,1^{*}$ & 0,18 \\
Hipo/hipertiroidismo & $17,7 \%$ & $78,8^{*}$ & 0,09 \\
\hline
\end{tabular}

Nota. ${ }^{\star}$ p 0,001 .

Entre los más jóvenes (15 a 29 años) los diagnósticos más frecuentes entre quienes consumen fármacos opiáceos fueron: dolores de cabeza o migraña $(42,7 \%)$, alergia crónica $(30,1 \%)$, dolor lumbar $(19,8 \%)$, asma $(18,5 \%)$, dolor cervical o dorsal (13,9\%), hipo/hipertiroidismo $(11,8 \%)$, depresión $(8,7 \%)$, hipercolesterolemia $(8,4 \%)$ y ansiedad $(5,1 \%)$.

Quienes están consumiendo opiáceos en el momento de la encuesta presentan hábitos sedentarios con mayor frecuencia que quienes no los consumen (16,5\% vs. $\left.12,0 \% ; \chi^{2}=10,9 ; \mathrm{p}<0,001 ; \mathrm{V}=0,04\right)$, presentan con mayor frecuencia obesidad $\left(22,2 \%\right.$ vs. $11,0 \% ; \chi^{2}=92,4 ; p<0,01$; $\mathrm{V}=0,10)$, fuman diariamente con mayor frecuencia $(21,6 \%$ vs. $\left.18,6 \% ; \chi^{2}=9,6 ; p<0,05 ; \mathrm{V}=0,03\right)$ y presentan consumo problemático de alcohol con menor frecuencia (9,8\% vs. $\left.16,9 \% ; \chi^{2}=27,1 ; \mathrm{p}<0,001 ; \mathrm{V}=0,06\right)$.

De entre quienes consumen analgésicos opiáceos, el 9,9\% lo hace sin prescripción médica. No hay diferencias por sexo entre quienes hacen un uso no prescrito de estos fármacos (hombres $11,0 \%$, mujeres $9,3 \% ; \chi^{2}=0,99 ; \mathrm{p}=$ $0,35)$. En cambio, sí hay diferencias significativas por grupo de edad: los más jóvenes (15 - 29 años) consumen opiáceos no prescritos en el 17,6\% de los casos, los de $30-44$ en el $13,9 \%$, los de 45 - 64 en el $8,6 \%$ y los de 65 o más en el 3,1\% ( $\left.\chi^{2}=39,5 ; \mathrm{p}<0,001 ; \mathrm{V}=0,17\right)$.

\section{Discusión}

Los datos del presente estudio muestran cifras llamativas de uso de analgésicos opiáceos en la población general.
Entre un 15 y un $17 \%$ ha utilizado estos fármacos en el último año y alrededor del $9 \%$ lo estaba haciendo en el momento de realización de la encuesta. Estas prevalencias superan las encontradas para todo el territorio español en el estudio EDADES 2017 (DGPNSD, 2018), en el que se encuentra que el consumo en el último año alcanza el 6,7\% y en el último mes el 2,9\%. Es muy probable que, como ya ha sucedido en otros países, el consumo de este tipo de medicamentos se produzca preferentemente en entornos urbanos, al menos en sus inicios, distribuyéndose progresivamente hacia las áreas rurales próximas (Keyes, Cerdá, Brady, Havens y Galea, 2014). Por lo demás, los hallazgos del EDADES coinciden con los del presente estudio en la mayor prevalencia en mujeres y el incremento de la prevalencia de consumo con la edad.

Es difícil concebir que casi un $10 \%$ de la población de una gran ciudad como Madrid presente patologías que justifiquen el uso de estos fármacos. En el caso de que estas patologías estuvieran presentes, debería esperarse una mayor frecuencia de uso de estos medicamentos para tratarlas en los grupos de edad más avanzada. Sin embargo, apenas existen diferencias entre los porcentajes de sujetos que los están tomando a partir de los 30 años hasta más allá de los 65. Pero más sorprendente aún resulta el hecho de que en torno al 12,5\% de quienes tienen más de 15 años y no han cumplido aún los 30 los hayan tomado en el último año y la mitad de ellos sigan tomándolos en el momento de la encuesta. Cuando repasamos los diagnósticos recibidos por este grupo de edad descubrimos, en primer lugar, los dolores de cabeza o migrañas, en más del $40 \%$. En el caso de la migraña los análgésicos opioides están contraindicados (Casucci y Cevoli, 2013; Tepper, 2012) y mucho más en dolores menos severos (DeVries, Koch, Wall, Getchius, Chi y Rosenberg, 2014). Los dolores de espalda a cualquier nivel no es muy probable que requieran tratamiento médico en todos los casos y difícil de explicar que, caso de requerirlo, deban prescribirse opiáceos (Fleming, Rabago, Mundt y Fleming, 2007; Sturgeon, 2014). Mucho más difícil de explicar es la relación entre el uso de estos fármacos y problemas como alergia, asma o hipercolesterolemia, entre otros.

La vinculación entre uso de analgésicos opiáceos y problemas de salud mental está bien documentada (Richardson et al., 2012). Los pacientes con trastornos de salud mental y abuso de sustancias tienen más probabilidades de recibir tratamiento opioide a largo plazo para el dolor crónico y más probabilidades de tener resultados adversos de esta terapia, disponiéndose de pocas pruebas del beneficio a largo plazo del tratamiento con opioides en personas con trastornos psicopatológicos (Edlund et al., 2010; Davis, Lin, Liu y Sites, 2017; Howe y Sullivan, 2014; Seal et al., 2012). Aunque es difícil establecer direcciones causales, existen indicios de que el uso crónico de opiáceos de prescripción puede causar o, cuando menos, agravar diferentes trastornos psicopatológicos (Becker, Sullivan, Tetrault, Desai y Fiellin, 2008). El 
riesgo de desarrollar una depresión aumenta a medida que aumenta la duración de la exposición a analgésicos opioides (Scherrer et al., 2014). En la muestra del presente estudio quienes están tomando analgésicos opiáceos han recibido el triple de diagnósticos de ansiedad y depresión y consumen simultáneamente el triple de fármacos antidepresivos y ansiolíticos. No es posible discriminar si esos diagnósticos favorecen la prescripción de fármacos opiáceos o es el uso agudo o crónico de estos analgésicos el factor que incrementa los síntomas ansiosodepresivos, pero queda fuera de toda duda la vinculación entre opiáceos y problemas de salud mental.

El uso de fármacos opiáceos en el presente estudio es más frecuente entre las mujeres (razón 2:1) y personas con nivel de estudios bajo y de clase social desfavorecida. Las diferencias por sexo son comunes en la mayor parte de los estudios, siendo sistemáticamente más frecuente el uso de estos fármacos en mujeres (Dale et al., 2015). Las mujeres informan de su experiencia de dolor con mayor frecuencia que los hombres, tienen mayores tasas de diagnósticos relacionados con el dolor, tienen mayor sensibilidad al dolor y tienen una respuesta variable al dolor y a la analgesia (Koons, Greenberg, Cannon y Beauchamp, 2018).

Casi un $10 \%$ de quienes dicen consumir estos fármacos a menudo lo hace sin prescripción médica, siendo más frecuente entre los más jóvenes. No es posible conocer mediante el presente estudio la forma de obtención de los analgésicos opiáceos no prescritos. Es posible que en buena medida se deba a la disponibilidad doméstica de este tipo de medicamentos en el "cajón de las medicinas" y que sean los propios familiares los que faciliten su uso al resto de convivientes, valorando la potencia analgésica sin consciencia de riesgos. También es posible que se esté configurando un mercado negro de estos medicamentos (que se pueden adquirir sin receta en Internet, p. ej., https://seasano.net/oxycodone), inducido por subculturas juveniles, como la desarrollada en torno al reggaeton y a Youtube, algunas de cuyas figuras han popularizado canciones sobre el "perco" (nombre en argot del Percocet, Oxicodona), cuyo uso ya es conocido en adolescentes madrileños de barrios periféricos y en la clínica de los centros específicos de tratamiento. Sin embargo, este fenómeno es tan reciente que sólo podemos movernos en el terreno de la mera especulación, pues, aunque abundan las noticias en prensa, aún carecemos de aproximaciones científicas valorables. Hay que tener en cuenta que en estudios previos son los más jóvenes los que pueden pasar con mayor facilidad del uso al abuso y multiplicar el riesgo de sobredosis (Nechuta, Tyndall, Mukhopadhyay y McPheeters, 2018). La Estrategia Nacional sobre Adicciones 2017- 2024, formulada por el Plan Nacional sobre Drogas (PNSD, 2018), apenas ha prestado atención a este riesgo potencial, que puede convertirse en un grave problema de salud pública en los próximos años.

El presente estudio cuenta con algunas limitaciones. Los datos se han obtenido a partir de tres preguntas sencillas, aportando una breve lista de medicamentos opiáceos. Es probable que quien identifique alguno de ellos responda afirmativamente, pero la lista no agota las posibles presentaciones comerciales que contienen estos preparados. Por ello, es posible que la frecuencia de uso encontrada sea menor que la real. Tampoco esta encuesta incluye a menores de 15 años, que pueden ser el grupo poblacional en mayor riesgo de comenzar a usar estos fármacos y habituarse a su uso, inicialmente normalizado, y a dar el salto al mercado negro, como sabemos que ha sucedido en otros contextos culturales. Sin embargo, la principal fortaleza del presente trabajo es el estudio de una muestra representativa de una población urbana, proporcionando por primera vez datos que permiten cuantificar inicialmente un problema y sugerir hipótesis más audaces en estudios futuros.

En conclusión, el presente estudio encuentra prevalencias elevadas de uso de fármacos opiáceos en la población de Madrid, difícilmente justificables por patologías que aconsejen su prescripción. Además, una parte significativa se consume al margen de la prescripción médica. Sin que pueda establecerse ningún paralelismo con la denominada "epidemia de opiáceos" en Estados Unidos, sí puede considerarse que podríamos estar en el umbral de problemas similares a los ya conocidos. Los estudios oficiales sobre prescripción de analgésicos opiáceos ya hace tiempo que alertan sobre el crecimiento sostenido de la prescripción médica de este tipo de fármacos y el presente estudio encuentra que existen sectores de población con especial vulnerabilidad (mujeres, jóvenes, de clase desfavorecida y bajo nivel cultural). Como ya sabemos por otros países, este problema y los que pueden derivarse son de naturaleza muy compleja, integrando factores bioquímicos, psicológicos, comerciales, culturales, políticos, legales, etc. En todo caso, este estudio complementa los datos oficiales disponibles y alerta a los expertos, las autoridades sanitarias y los profesionales a tomar en consideración lo que podría ser el germen de un problema de enorme gravedad, que en modo alguno debería alcanzarnos por sorpresa, al tener modelos bien conocidos y estudiados en otros países.

\section{Conflicto de intereses}

Los autores declaran no presentar ningún conflicto de intereses.

\section{Agradecimientos}

Los autores quieren agradecer a cada una de las personas que han colaborado en la realización de este estudio: Alejandro Blanco Quintana, Gema Blasco Novalbos; Nieves Botella Cañamares, Yolanda Quintana Moreno; José Manuel Díaz Olalla, M ${ }^{\text {a }}$ Rosario Sanz Cuesta, Isabel Junco Torres, Carmen López Jiménez; y Mercedes Rodríguez Pérez, todos ellos de Madrid Salud. 


\section{Referencias}

AEMPS (2017). Utilización de medicamentos opioides en España durante el periodo 2008-2015. Ministerio de Sanidad, Servicios Sociales e Igualdad. Recuperado de https:// www.aemps.gob.es/medicamentosUsoHumano/observatorio/docs/opioides-2008-2015.pdf.

AEMPS (2019). Utilización de medicamentos opioides en Espana durante el periodo 2010-2017. Recuperado de https:/ / www.aemps.gob.es/medicamentosUsoHumano/observatorio/informes-publicados/informes-opioides-espana-2010-2017.htm.

Ainsworth, B. E., Haskell, W. L., Whitt, M. C., Irwin, M. L., Swartz, A. M., Strath, S. J.,... Jacobs, D. R. (2000). Compendium of physical activities: An update of activity codes and MET intensities. Medicine and Science in Sports and Exercise, 32, S498-S504. doi:10.1097/00005768200009001-00009.

Ashburn, M. A. y Fleisher, L. A. (2018). Increasing evidence for the limited role of opioids to treat chronic noncancer pain. JAMA, 320, 2427-2428. doi:10.1001/ jama.2018.19327.

Alonso Babarro, A., Varela Cerdeira, M. y Aparicio Jabalquinto, G. (2003). Evolución del consumo de opioides en un área de Madrid desde 1997 a 2001. Atención Primaria, 32, 390-392.

Becker, W. C., Sullivan, L. E., Tetrault, J. M., Desai, R. A. y Fiellin, D. A. (2008). Non-medical use, abuse and dependence on prescription opioids among US adults: Psychiatric, medical and substance use correlates. Drug and Alcohol Dependence, 94, 38-47. doi:10.1016/j.drugalcdep.2007.09.018.

Busse, J. W., Craigie, S., Juurlink, D. N., Buckley, D. N., Wang, L., Couban, R. J.,... Cull, C. (2017). Guideline for opioid therapy and chronic noncancer pain. CMAJ, 189, 659-666. doi:10.1503/cmaj.170363.

Calabozo Freile, B. (2017). Opioides: Del poco uso ... al abuso. El Ojo de Markov, 59. Recuperado de https:// www.saludcastillayleon.es/portalmedicamento/es/ cim-sacyl/ojo-markov/opioides-uso-abuso.

Casucci, G. y Cevoli, S. (2013). Controversies in migraine treatment: Opioids should be avoided. Neurological Sciences, 34, 125-128. doi:10.1007/s10072-013-1395-8.

Dale, O., Borchgrevink, P. C., Fredheim, O. M. S., Mahic, M., Romundstad, P. y Skurtveit, S. (2015). Prevalence of use of non-prescription analgesics in the Norwegian HUNT3 population: Impact of gender, age, exercise and prescription of opioids. BMC Public Health, 15, 461. doi:10.1186/s12889-015-1774-6.

Davis, M. A., Lin, L. A., Liu, H. y Sites, B. D. (2017). Prescription opioid use among adults with mental health disorders in the United States. Journal of the American Board of Family Medicine, 30, 407-417. doi:10.3122/jabfm.2017.04.170112.
DeVries, A., Koch, T., Wall, E., Getchius, T., Chi, W. y Rosenberg, A. (2014). Opioid use among adolescent patients treated for headache. Journal of Adolescent Health, 55, 128-133. doi:10.1016/j.jadohealth.2013.12.014.

DGPNSD. (2018). Edades 2017. Encuesta sobre alcohol y otras drogas en España (EDADES), 1995-2017. Madrid: Ministerio de Sanidad, Consumo y bienestar Social; Delegación del Gobierno para el Plan Nacional sobre Drogas. Recuperado de http://www.pnsd.mscbs.gob.es/profesionales/sistemasInformacion/sistemaInformacion/pdf/ EDADES_2017_Informe.pdf.

Díaz Olalla, J. M. y Benítez Robredo, M. T. (2015). Estudio de salud de la ciudad de Madrid, 2014. Madrid: Madrid Salud. Recuperado de http://www.madridsalud.es/publicaciones/OtrasPublicaciones/EstudioSaludCiudadMadrid.pdf.

Domingo-Salvany, A., Bacigalupe, A., Carrasco, J. M., Espelt, A., Ferrando, J. y Borrell, C. (2013). Propuestas de clase social neoweberiana y neomarxista a partir de la Clasificación Nacional de Ocupaciones 2011. Gaceta Sa nitaria, 27, 263-272. doi:10.1016/j.gaceta.2012.12.009.

Dowell D., Haegerich T.M. y Chou, R. (2016). Guideline for Prescribing Opioids for Chronic Pain - United States, 2016. Recommendations and Reports, 65, 1-49. doi:10.15585/mmwr.rr6501e1.

Edlund, M. J., Martin, B. C., Devries, A., Fan, M. Y., Braden, J. B. y Sullivan, M. D. (2010). Trends in use of opioids for chronic non-cancer pain among individuals with mental health and substance use disorders: The TROUP study. Clinical Journal of Pain, 26, 1-8. doi:10.1097/AJP.0b013e3181b99f35.

Els, C., Jackson, T. D., Kunyk, D., Lappi, V. G., Sonnenberg, B., Hagtvedt, R.,... Straube S. (2017). Adverse events associated with medium- and long-term use of opioids for chronic non-cancer pain: An overview of Cochrane Reviews. Cochrane Database of Systematic Reviews, 10, CD012509. doi:10.1002/14651858.CD012509.pub2.

Fleming, S., Rabago, D. P., Mundt, M. P. y Fleming, M. F. (2007). CAM therapies among primary care patients using opioid therapy for chronic pain. BMC Complementary and Alternative Medicine, 7, 15. doi:10.1186/14726882-7-15.

Gaspari, S., Papachatzaki, M.M., Koo, J.W., Carr, F.B., Tsimpanouli, M.E., Stergiou, E.,... Zachariou, V. (2014). Nucleus accumbens-specific interventions in rgs9-2 activity modulate responses to morphine. Neuropsychopharmacology, 39, 1968-1977. doi:10.1038/npp.2014.45.

Goldberg, D. P. y Williams, P. (1998). A user's guide to the General Health Questionnaire. London: Nfer-Nelson.

Guardia Serecigni, J. (2018). Overdose epidemic linked to the prescription of opioid analgesics in the United States. Adicciones, 30, 87-92. doi:10.20882/adicciones.936.

Hadland, S. E., Cerdá, M., Li, Y., Krieger, M. S. y Marsha11, B. D. (2018). Association of pharmaceutical industry 
marketing of opioid products to physicians with subsequent opioid prescribing. JAMA Internal Medicine, 178, 861-863. doi:10.1001/jamanetworkopen.2018.1999.

Hadland, S. E., Rivera-Aguirre, A., Marshall, B. D. y Cerdá, M. (2019). Association of pharmaceutical industry marketing of opioid products with mortality from opioid-related overdoses. JAMA Network Open, 2, e186007. doi:10.1001/jamanetworkopen.2018.6007.

Howe, C. Q. y Sullivan, M. D. (2014). The missing 'P' in pain management: How the current opioid epidemic highlights the need for psychiatric services in chronic pain care. General Hospital Psychiatry, 36, 99-104. doi:10.1016/j.genhosppsych.2013.10.003.

Keyes, K. M., Cerdá, M., Brady, J. E., Havens, J. R. y Galea, S. (2014). Understanding the rural-urban differences in nonmedical prescription opioid use and abuse in the United States. American Journal of Public Health, 104, 5259. doi:10.2105/AJPH.2013.301709.

Kolodny, A., Courtwright, D. T., Hwang, C. S., Kreiner, P., Eadie, J. L., Clark, T. W. y Alexander, G. C. (2015). The prescription opioid and heroin crisis: A public health approach to an epidemic of addiction. Annual Review of Public Health, 36, 559-574. doi:10.1146/annurev-publhealth-031914-122957.

Koons, A. L., Greenberg, M. R., Cannon, R. D. y Beauchamp, G. A. (2018). Women and the experience of pain and opioid use disorder: A literature-based commentary. Clinical Therapeutics, 40, 190-196. doi:10.1016/j. clinthera.2017.12.016.

Lavand'homme, P. y Steyaert, A. (2017). Opioid-free anesthesia opioid side effects: Tolerance and hyperalgesia. Best Practice \& Research Clinical Anaesthesiology, 31, 487498. doi:10.1016/j.bpa.2017.05.003.

Lizán, L. y Reig, A. (1999). Adaptación transcultural de una medida de la calidad de vida relacionada con la salud: La versión española de las viñetas COOP/WONCA. Atención Primaria, 24, 75-82.

Marshall, B., Bland, M. K., Hulla, R. y Gatchel, R. J. (2019). Considerations in addressing the opioid epidemic and chronic pain within the USA. Pain Management, 9, 131138. doi:10.2217/pmt-2018-0070.

Merskey, H. (1986). International Association for the Study of Pain (IASP): Subcommittee on taxonomy, classification of chronic pain, description of pain terms. Pain, 3, S1-S226.

Nechuta, S. J., Tyndall, B. D., Mukhopadhyay, S. y McPheeters, M. L. (2018). Sociodemographic factors, prescription history and opioid overdose deaths: A statewide analysis using linked PDMP and mortality data. Drug and Alcohol Dependence, 190, 62-71. doi:10.1016/j.drugalcdep.2018.05.004.

Pedrero-Pérez, E. J. y Díaz-Olalla, J. M. (2016). COOP/ WONCA: Fiabilidad y validez de la prueba adminis- trada telefónicamente. Atención Primaria, 48, 25-32. doi:10.1016/j.aprim.2014.12.010.

PNSD. (2018). Estrategia Nacional sobre Adicciones 20172024. Madrid: Plan Nacional sobre Drogas, Ministerio de Sanidad, Servicios Sociales e Igualdad. Recuperado de https://cendocps.carm.es/documentacion/2018_ Estrategia_Nacional_Adicciones_2017-2024.pdf.

Randall, C. y Crane, J. (2014). Tramadol deaths in Northern Ireland: A review of cases from 1996 to 2012. Jour nal of Forensic and Legal Medicine, 23, 32-36. doi:10.1016/j. jflm.2014.01.006.

Richardson, L. P., Russo, J. E., Katon, W., McCarty, C. A., DeVries, A., Edlund, M. J.,... Sullivan, M. (2012). Mental health disorders and long-term opioid use among adolescents and young adults with chronic pain. Journal of Adolescent Health, 50, 553-558. doi:10.1016/j.jadohealth.2011.11.011.

Rocha, K. B., Pérez, K., Rodríguez-Sanz, M., Borrell, C. y Obiols. J. E. (2011). Propiedades psicométricas y valores normativos del General Health Questionnaire (GHQ12) en población general española. International Journal of Clinical and Health Psychology, 11, 125-139.

Rosenquist R. (2019). Use of opioids in the management of chronic non-cancer pain. UpToDate. Recuperado de https:/ / www.uptodate.com/contents/use-of-opioids-inthe-management-of-chronic-non-cancer-pain.

Ruiz-López, D. y Alonso-Babarro, A. (2019). Consumo de opioides en la Comunidad de Madrid (España) entre 2004 y 2014. Revista Clínica Española, 219, 367-374. doi:10.1016/j.rce.2019.02.002.

Scherrer, J. F., Svrakic, D. M., Freedland, K. E., Chrusciel, T., Balasubramanian, S., Bucholz, K. K.,... Lustman, P. J. (2014). Prescription opioid analgesics increase the risk of depression. Journal of General Internal Medicine, 29, 491-499. doi:10.1007/s11606-013-2648-1.

Seal, K. H., Shi, Y., Cohen, G., Cohen, B. E., Maguen, S., Krebs, E. E. y Neylan, T. C. (2012). Association of mental health disorders with prescription opioids and high-risk opioid use in US veterans of Iraq and Afghanistan. JAMA, 307, 940-947. doi:10.1001/jama.2012.234.

Skolnick, P. (2018). The opioid epidemic: Crisis and solutions. Annual Review of Pharmacology and Toxicology, 58, 143159. doi:10.1146/annurev-pharmtox-010617-052534.

Socidrogalcohol (2017). Guía de consenso para el buen uso de analgésicos opioides. Gestión de riesgos y beneficios. Valencia: Socidrogalcohol.

Sturgeon, J. A. (2014). Psychological therapies for the management of chronic pain. Psychology Research and Behavior Management, 7, 115-124. doi:10.2147/PRBM.S44762.

Tepper, S. J. (2012). Opioids should not be used in migraine. Headache, 52, 30-34. doi:10.1111/j.15264610.2012.02140.x. 